\title{
Los componentes del pastoreo afectan de forma diferencial las micorrizas en Paspalum dilatatum Poir.
}

\author{
Silvina García ${ }^{1}$; Fabiana Pezzani ${ }^{1}{ }^{\bowtie}$; Felipe Lezama ${ }^{1}$ \& José M. Paruelo ${ }^{2}$ \\ ${ }^{1}$ Ecología. Departamento de Sistemas Ambientales. Facultad de Agronomía. Universidad de la República. Montevideo, \\ Uruguay. ${ }^{2}$ Grupo de Ecología de Pastizales (GEP), Instituto de Ecología y Ciencias Ambientales, Facultad de Ciencias, \\ Universidad de la República. Montevideo, Uruguay. Instituto Nacional de Investigación Agropecuaria. Estación Experimental \\ La Estanzuela, Colonia, Uruguay. Cátedra de Ecología. IFEVA, Facultad de Agronomía, IFEVA. Universidad de Buenos Aires \\ y CONICET. Buenos Aires, Argentina.
}

\begin{abstract}
RESUMEN. El pastoreo es una perturbación importante en los ecosistemas de pastizales, ya que altera su estructura y su funcionamiento. Las micorrizas arbusculares son interacciones cosmopolitas de estos ecosistemas, y se destacan por su incidencia elevada en las gramíneas. Las micorrizas muestran respuestas diversas y contradictorias al pastoreo (ser beneficiadas, perjudicadas o no ser afectadas). Una posible hipótesis es que cada componente del pastoreo (defoliación selectiva, deyecciones y pisoteo) diferiría en su importancia relativa al afectar la micorrización. El objetivo del presente trabajo fue evaluar el efecto del pisoteo, la selectividad y la deposición de orina sobre las micorrizas de Paspalum dilatatum, una de las principales gramíneas nativas de los pastizales del Río de la Plata. En un experimento manipulativo en el que se simularon los distintos componentes del pastoreo se evaluó el efecto de cada componente de forma aislada y en interacción sobre la colonización micorrícica total, así como de las diferentes estructuras fúngicas (arbúsculos, ovillos y vesículas). La colonización micorrícica total y por arbúsculos no fueron afectadas por ninguno de los componentes del pastoreo, mientras que sí se observaron efectos sobre las demás estructuras fúngicas. La colonización por ovillos (una de las estructuras que participan en el intercambio con el hospedante) fue afectada de forma positiva por la selectividad y negativa por el agregado de nitrógeno (simulando la deposición de orina); estas estructuras también fueron afectadas por las interacciones selectividad $\mathrm{x}$ pisoteo y pisoteo $\mathrm{x}$ agregado de $\mathrm{N}$. El pisoteo afectó negativamente la presencia de vesículas, lo que podría deberse, en parte, al daño mecánico que esta perturbación causa tanto sobre las raíces como sobre el micelio extra-radical. El estudio de los componentes del pastoreo de manera aislada mostró que cada uno ejerce efectos diferentes sobre las micorrizas, y su importancia relativa podría afectar el resultado global del pastoreo sobre esta interacción.
\end{abstract}

[Palabras clave: micorrizas arbusculares, defoliación selectiva, deposición de orina, pisoteo]

\begin{abstract}
Aвstract. Grazing components differentially affect mycorrhizas in Paspalum ditatatum Poir. Grazing by large herbivores represents an important disturbance in natural grasslands, affecting its structure and function. Arbuscular mycorrhizas (AM) are cosmopolitan interactions in these ecosystems, particularly in grasses, main constituent of this ecosystem. Mycorrhizas show contradictory responses to grazing (AM colonization has been positively, negatively and not affected by grazing). We hypothesized that each grazing component (selective defoliation, trampling and urine deposition) would differentially affect mycorrhizal colonization, and this could explain the high variability in AM responses. Our objective was to assess the effect of trampling, selective defoliation and nitrogen $(\mathrm{N})$ addition on AM colonization of one of the most important grasses in Río de la Plata grasslands: Paspalum dilatatum. Grazing components were simulated in a manipulative experiment where we evaluated their individual and combined effects on total AM colonization and the occurrence of different fungal structures (arbuscules, coils and vesicles). No grazing component affected total AM colonization and arbuscules, while other fungi structures were affected by different treatments. Coil colonization (one of the nutrient interchange structures between the symbionts) was positively affected by selective defoliation and it was negatively affected by $\mathrm{N}$ addition; these structures were also affected by the combined effect between selectivity $\mathrm{x}$ trampling and by trampling $\mathrm{x} \mathrm{N}$ addition. Vesicle colonization was negatively affected by trampling, maybe because of mechanical damage to roots and to extraradical mycelium caused by this disturb. The study of the individual grazing components showed that each one caused different effects on mycorrhizal interaction and their relative influence could affect the overall grazing effect on this interaction.
\end{abstract}

[Keywords: arbuscular mycorrhiza, selective defoliation, urine deposition, trampling]

Editora asociada: Marina Omacini

\abiana@fagro.edu.uy
Recibido: 20 de Agosto de 2018

Aceptado: 5 de Enero de 2019 


\section{INTRODUCCIÓN}

Los efectos del pastoreo por grandes herbívoros en áreas de pastizal dan lugar a cambios no sólo en la vegetación (McNaughton 1984; Altesor et al. 2006), sino también en los microorganismos del suelo y en sus interacciones con las plantas, como las asociadas a las micorrizas arbusculares (Gehring and Whitham 1994; Barto and Rillig 2010; Bai et al. 2013; van der Heyde et al. 2017). La interacción micorrícica arbuscular es el tipo más extendido y frecuente en la naturaleza. Entre los beneficios que esta interacción proporciona a las plantas se destacan el aumento en la disponibilidad de nutrientes, especialmente fósforo (Smith and Smith 2011), y de agua (Marulanda et al. 2003), así como la protección contra microorganismos patógenos (Schausberger et al. 2012). En particular, esta interacción es clave en los pastizales dada su gran incidencia en gramíneas (principales constituyentes de estos ecosistemas), en especial sobre aquellas especies con metabolismo fotosintético $\mathrm{C}_{4}$ (Hartnett and Wilson 1999; Pezzani et al. 2006; Endresz et al. 2013; García et al. 2016). En estas interacciones, las hifas de los hongos micorrizógenos arbusculares (HMA) penetran las células corticales de las raíces de las plantas y forman estructuras características: arbúsculos (estructuras que resultan de la ramificación de las hifas y cuya función es el intercambio metabólico entre simbiontes), vesículas (estructuras globosas con función de almacenamiento de reservas para los hongos) y ovillos que se forman a partir de hifas que adquieren conformación torneada dentro de la célula cortical (Dickson et al. 2007; Smith and Read 2008). Tanto los arbúsculos como los ovillos participan en el intercambio con la planta hospedante (Hong et al. 2012) y representan variaciones morfológicas de la colonización. Smith y Read (2008) y Hong et al. (2012) plantean que los arbúsculos y los ovillos podrían corresponder a diferentes taxones de hongos que estarían colonizando las raíces de la planta. La presencia de cada una de las estructuras fúngicas en las raíces de las plantas, medida como porcentaje, se denomina intensidad de colonización micorrícica y es un indicador del grado de funcionamiento de dicha interacción, es decir del intercambio metabólico entre los simbiontes (Smith and Read 2008). Además de la colonización intra-radical, para evaluar los efectos del mutualismo se utiliza la presencia de estructuras fúngicas extra-radicales (Collins et al. 2003) y atributos sobre el desempeño de las plantas (Smith and Smith 2011).

Los antecedentes acerca de los efectos del pastoreo sobre la intensidad de colonización micorrícica muestran una gran variación y con signos opuestos. Por ejemplo, Grigera y Oesterheld (2004) en su trabajo llevado a cabo en la Pampa inundable, y Bai et al. (2013) en su estudio en los pastizales de Mongolia, hallaron que tanto la intensidad como la duración del pastoreo causaron efectos negativos sobre las micorrizas arbusculares. Lugo et al. (2003) y van der Heyde et al. (2017) reportaron que la intensidad de colonización por micorrizas no se vio afectada por el pastoreo en sistemas de pastizales montañosos. Por otra parte, Wallace (1981), Eom et al. (2001) y Guo et al. (2016) hallaron efectos positivos del pastoreo sobre la micorrización en pastizales del Serengeti, Kansas y Mongolia, respectivamente. Dos revisiones que analizaron varios antecedentes en los que se evaluaron los impactos de la herbivoría sobre la colonización por HMA arrojaron resultados diferentes y contradictorios (Gehring and Whitham 1994; Barto and Rillig 2010). Por un lado, Gehring y Whitham (1994) hallaron efectos negativos de la herbivoría sobre la micorrización en 23 especies vegetales de un total de 37 estudiadas y explicaron dicha respuesta mediante la hipótesis de limitación por carbono (C). La misma postula que la remoción de tejido fotosintético provocada por el pastoreo implica un decremento en el transporte de C hacia las raíces, lo que reduce la energía disponible para el crecimiento de los HMA. En cambio, Barto y Rillig (2010), a partir de un meta-análisis de 33 publicaciones acerca de los efectos de la herbivoría sobre la colonización micorrícica, concluyeron que la misma tendría un efecto poco marcado sobre dicha interacción. Algunos autores buscaron explicar las diferentes respuestas de las micorrizas a la herbivoría a través del estudio de factores tales como la composición florística (Mendoza et al. 2002; Ruotsalainen and Eskelinen 2011), la fertilidad del suelo (Mendoza et al. 2002; Guo et al. 2016) y el estadío fenológico de las plantas (Piippo et al. 2011).

La inconsistencia en las respuestas de las micorrizas al pastoreo podría reflejar el hecho de que la mayoría de los estudios realizados tomaron al pastoreo en forma global. Aunque escasos, existen trabajos que evaluaron los efectos de alguno de los componentes del pastoreo sobre las micorrizas 
arbusculares. Antecedentes sobre el efecto de la defoliación experimental en gramíneas no revelaron efectos de este componente del pastoreo sobre la presencia de los HMA (Walling and Zabinski 2006; Torres et al. 2011). El efecto del agregado de nitrógeno $(\mathrm{N}$, uno de los principales componentes de la orina) mostró un aumento de la diversidad de hongos micorrizógenos (Avio et al. 2013), así como también efectos sobre la estructura de las comunidades micorrícicas en un pastizal semiárido de Nuevo México (PorrasAlfaro et al. 2007). Hiltbrunner et al. (2012) estudiaron el efecto de diferentes intensidades de pisoteo y observaron una reducción en la abundancia de los microorganismos del suelo, particularmente acentuada en el grupo de los hongos. Cabe destacar que ninguno de los antecedentes evaluó el efecto de todos los componentes del pastoreo en forma simultánea, sino que se enfocaron en alguno de ellos de manera aislada sin considerar que el pastoreo se trata de un proceso complejo en el que sus distintos componentes (pisoteo, selectividad, intensidad de la remoción, deposición de heces y orina) se encuentran en interacción.

Paspalum dilatatum es una gramínea frecuente en los pastizales del Río de la Plata (León 1991), de gran palatabilidad y calidad forrajera (Bungenstab 2009), con metabolismo fotosintético $\mathrm{C}_{4}$ y generalmente con un grado de micorrización elevado (García et al. 2016). En relación con el pastoreo, esta gramínea se comportaría como creciente al pastoreo (Altesor et al. 1998), mientras que respecto a los componentes, Lezama y Paruelo (2016) reportaron que fue favorecida por el pisoteo y por la interacción entre el agregado de $\mathrm{N}$ y la defoliación selectiva. En cuanto al efecto del pastoreo sobre la micorrización en $P$. dilatatum, Grigera y Oesterheld (2004) observaron que dicha interacción fue afectada de manera negativa por el pastoreo en la mayor parte del año. En cambio, en una comunidad de pastizal del centro-sur de Uruguay, el pastoreo no afectó la colonización total, pero sí afectó negativamente la presencia de ovillos, una de las estructuras de intercambio de la interacción (datos no publicados).

El objetivo del presente trabajo fue evaluar el efecto de tres componentes del pastoreo (pisoteo, selectividad en la remoción de biomasa y deposición de orina) sobre la micorrización de $P$. dilatatum. De esta manera, se buscó explicar parte de la variación observada en las respuestas de la micorrización al pastoreo a través del análisis de los componentes aislados que involucra este disturbio. Para cumplir con este objetivo se contó con un experimento en campo establecido en 2006 por Lezama y Paruelo (2016) que evaluó el efecto de la defoliación selectiva, el pisoteo y la deposición de orina (simulado mediante el agregado de N) sobre la vegetación de un pastizal natural en la región centro-sur de Uruguay. Este ensayo representó una excelente oportunidad para poder evaluar también el efecto de los componentes del pastoreo (aislados e interactuando) sobre la interacción micorrícica. Sobre la base de antecedentes, postulamos que los distintos componentes del pastoreo podrían tener efectos contrapuestos y de diferente magnitud sobre las micorrizas.

\section{Materiales y Métodos}

\section{Sitio de estudio}

El presente trabajo se realizó en un área del establecimiento privado "El Relincho" (departamento de San José, Uruguay; 34¹9' S - 57 $02^{\prime} \mathrm{O}$ ) destinada a la ganadería extensiva con vacunos y ovinos. El ensayo se instaló en el año 2006 en un área excluida al pastoreo (por detalles, ver Lezama and Paruelo 2016), localizada sobre suelos de tipo vertisol rúptico (Altamirano et al. 1976). Los suelos donde se llevó a cabo el ensayo presentaron valores promedio de $7.25 \%$ de materia orgánica, $4.2 \%$ de carbono orgánico y 6.9 de pH. Se trata de un sitio de campo natural (pastizal) sin fertilización previa, por lo cual, de acuerdo con Hernández et al. (1995), son suelos pobres en fósforo.

\section{Diseño experimental}

El experimento consistió en un diseño factorial en bloques. En parcelas de 1.6x1.6 m se aplicaron cuatro tratamientos que simularon los diferentes componentes del pastoreo. Cada tratamiento presentó dos niveles: remoción de biomasa intensiva y moderada (simulado a través de cortes con tijera); remoción de biomasa selectiva y no selectiva (simulado a través de corte de toda la biomasa en pie en el caso del no selectivo y en el caso del selectivo no se cosecharon las especies que el ganado rechaza); parcelas pisoteadas y no pisoteadas (simulado mediante la aplicación estacional de una pezuña artificial de acuerdo al protocolo establecido por Striker et al. 2006); parcelas con y sin agregado de N (simulando la deposición 
de orina, según la dosis sugerida por Stillwell and Woodmansee [1981]). Cada uno de estos tratamientos tuvocuatro repeticiones. Además, se contó con cuatro parcelas de referencia, sin tratamiento. Lezama y Paruelo (2016) hallaron que la intensidad en la remoción de biomasa mostró escasos efectos sobre la frecuencia de las principales especies estudiadas, particularmente sobre $P$. dilatatum, por lo que consideramos que dicho factor podría tener poca relevancia a la hora de explicar las respuestas de la micorrización en esta gramínea. Por este motivo, para el presente trabajo se seleccionaron las combinaciones de tratamientos que incluían sólo la remoción intensiva de biomasa (consistente en la cosecha de $300 \mathrm{~g}$ de biomasa fresca en la parcela). De esta forma, los factores evaluados fueron el pisoteo $(\mathrm{P})$, el agregado de nitrógeno $(\mathrm{N})$ y la selectividad (S). Los ocho tratamientos resultantes de la combinación de los tres factores (P, N y S) fueron los siguientes: no selectivo (NoS), no selectivo con agregado de $\mathrm{N}$ (NoSN), no selectivo con agregado de $\mathrm{N}$ y pisoteado (NoSNP), no selectivo pisoteado (NoSP), selectivo (S), selectivo con agregado de $\mathrm{N}(\mathrm{SN})$, selectivo pisoteado (SP) y selectivo con agregado de $\mathrm{N}$ y pisoteado (SNP), lo que resultó en un total de 36 parcelas experimentales. Desde diciembre 2006 y hasta diciembre 2009 se evaluó la composición y la estructura de la comunidad vegetal, así como variables edáficas en respuesta a los componentes del pastoreo (Lezama and Paruelo 2016).

\section{Muestreo de raíces}

Tres años después de iniciados los tratamientos, en marzo de 2010, se realizó un muestreo de raíces de $P$. dilatatum en las 36 parcelas que formaron parte de este trabajo. En cada parcela se seleccionaron al azar tres individuos de $P$. dilatatum. Las raíces fueron separadas de la parte aérea y conservadas en vinagre puro para su posterior procesamiento en el laboratorio (Vierheilig et al. 1998).

\section{Procesamiento de las raíces para estudiar las micorrizas}

Las raíces fueron lavadas y aclaradas con una solución de $\mathrm{KOH}$ al $10 \%$, y luego se las tiñó con azul de tripano $(0.05 \%)$ según el método de Koske y Gemma (1989). Para cada individuo seleccionado en el campo se montaron en portaobjetos 30 segmentos de raíz de $1 \mathrm{~cm}$ de largo. Mediante observación al microscopio se identificaron estructuras típicas de los HMA: hifas, vesículas, arbúsculos y ovillos. Para cada individuo se cuantificó la intensidad de colonización total, estimada como el cociente entre el número de campos con presencia de alguna estructura fúngica y el número de campos observados $(n=90)$ (Brundrett 1996). También se calculó la intensidad de colonización para cada una de las siguientes estructuras: vesículas, arbúsculos y ovillos. Todas las variables fueron expresadas en porcentaje (promedio \pm error estándar).

\section{Procesamiento de datos y análisis estadísticos}

El análisis de los datos se realizó a través de un Modelo Lineal Generalizado Mixto (MLGM), familia Quasi Binomial y función de enlace logit. Los factores considerados en el modelo fueron la selectividad en la remoción de biomasa (S), el pisoteo (P) y el agregado de $\mathrm{N}(\mathrm{N})$. Las variables de respuesta fueron la colonización total, la colonización por vesículas y la colonización por cada una de las estructuras de intercambio (arbúsculos y ovillos). Las diferencias entre medias fueron analizadas con la prueba de diferencia mínima significativa (LSD, por su sigla en inglés), y se aplicó el ajuste por Bonferroni, con un valor de significancia de 0.05 , utilizando el programa INFOSTAT (Di Rienzo et al. 2014).

\section{Resultados}

La intensidad de colonización micorrícica total en $P$. dilatatum no fue afectada por ninguno de los componentes del pastoreo ni por sus interacciones (Tabla 1), al tiempo que presentó altos niveles en todos los tratamientos evaluados, $97.8 \pm 0.03 \%$ (promedio \pm error estándar). En todos los tratamientos se pudo observar la presencia de las diferentes estructuras fúngicas, siendo los valores de intensidad de colonización (\%, promedio \pm error estándar) de $3.3 \pm 0.01$ en el caso de los arbúsculos, $6.3 \pm 0.03$ para los ovillos y $42.2 \pm 0.04$ para las vesículas.

Al igual que la colonización total, la presencia de arbúsculos tampoco fue afectada significativamente por ninguno de los componentes del pastoreo evaluados ni por sus interacciones (Tabla 1). Sin embargo, los ovillos y las vesículas respondieron de manera diferencial a los distintos componentes del pastoreo. La colonización por ovillos fue afectada positivamente por la selectividad y negativamente por el agregado de $\mathrm{N}$. 
Tabla 1. Resultados del Modelo Lineal Generalizado Mixto con los componentes del pastoreo analizados (P: pisoteo, S: selectividad y N: agregado de nitrógeno) y sus interacciones sobre cada variable de la colonización micorrícica arbuscular en Paspalum dilatatum. Valores con asteriscos indican diferencias significativas $(P<0.05)$.

Table 1. Results of the Generalized Linear Mixed Model with grazing components (P: trampling, S: selective defoliation and $\mathrm{N}$ : nitrogen addition) and their interactions on arbuscular mycorrhizal colonization in Paspalum dilatatum. Asterisks denote significant differences $(P<0.05)$.

\begin{tabular}{lccccccc}
\hline Variable & \multicolumn{7}{c}{ Componentes del pastoreo } \\
& $\mathrm{S}$ & $\mathrm{P}$ & $\mathrm{N}$ & SxP & $\mathrm{PxN}$ & SxN & SxPxN \\
\hline Colonización micorrícica total & $\mathrm{P}=0.10$ & $\mathrm{P}=0.65$ & $\mathrm{P}=0.94$ & $\mathrm{P}=0.76$ & $\mathrm{P}=0.96$ & $\mathrm{P}=0.13$ & $\mathrm{P}=0.67$ \\
& $\mathrm{~F}=2.88$ & $\mathrm{~F}=0.21$ & $\mathrm{~F}=0.01$ & $\mathrm{~F}=0.09$ & $\mathrm{~F}=0.02$ & $\mathrm{~F}=2.49$ & $\mathrm{~F}=0.18$ \\
& $\mathrm{P}=0.75$ & $\mathrm{P}=0.76$ & $\mathrm{P}=0.32$ & $\mathrm{P}=0.90$ & $\mathrm{P}=0.53$ & $\mathrm{P}=0.57$ & $\mathrm{P}=0.07$ \\
Colonización por arbúsculos & $\mathrm{F}=0.11$ & $\mathrm{~F}=0.09$ & $\mathrm{~F}=1.03$ & $\mathrm{~F}=0.03$ & $\mathrm{~F}=0.09$ & $\mathrm{~F}=0.24$ & $\mathrm{~F}=6.61$ \\
& $\mathrm{P}=0.0003^{*}$ & $\mathrm{P}=0.57$ & $\mathrm{P}=0.0050^{*}$ & $\mathrm{P}=0.0362^{*}$ & $\mathrm{P}=0.0424^{*}$ & $\mathrm{P}=0.12$ & $\mathrm{P}=0.17$ \\
Colonización por ovillos & $\mathrm{F}=18.16$ & $\mathrm{~F}=0.34$ & $\mathrm{~F}=9.32$ & $\mathrm{~F}=4.92$ & $\mathrm{~F}=4.57$ & $\mathrm{~F}=2.54$ & $\mathrm{~F}=2.01$ \\
& $\mathrm{P}=0.85$ & $\mathrm{P}=0.0017^{*}$ & $\mathrm{P}=0.68$ & $\mathrm{P}=0.96$ & $\mathrm{P}=0.62$ & $\mathrm{P}=0.45$ & $\mathrm{P}=0.60$ \\
Colonización por vesículas & $\mathrm{F}=0.04$ & $\mathrm{~F}=12.48$ & $\mathrm{~F}=0.18$ & $\mathrm{~F}=0$ & $\mathrm{~F}=0.25$ & $\mathrm{~F}=0.58$ & $\mathrm{~F}=0.28$ \\
\hline
\end{tabular}

Estas estructuras no mostraron un efecto significativo del pisoteo en forma aislada (Tabla 1), sin embargo fueron afectadas por las interacciones selectividad $\mathrm{x}$ pisoteo $\mathrm{y}$ pisoteo $x$ agregado de $\mathrm{N}$ (Tabla 1). En presencia de pisoteo, la selectividad no mostró efecto sobre la colonización por ovillos. Pero en ausencia de pisoteo y con corte selectivo de biomasa, las raíces de $P$. dilatatum presentaron valores cinco

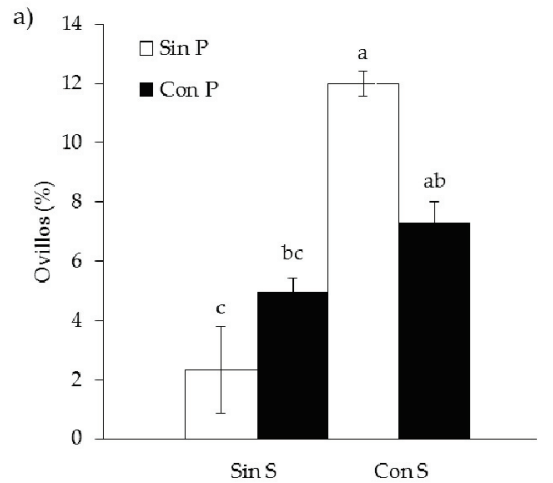

b)

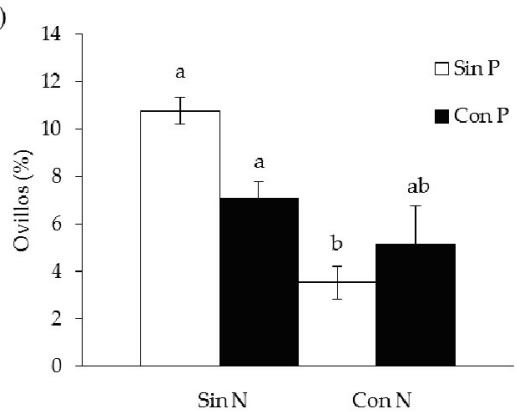

Figura 1. Efecto de los componentes del pastoreo sobre la intensidad de colonización por ovillos (\%, promedio \pm error estándar; $\mathrm{n}=4$ ) en Paspalum dilatatum: a) S: selectividad y P: pisoteo; b) P: pisoteo y N: agregado de nitrógeno. Letras diferentes indican diferencias significativas entre los tratamientos $(P<0.05)$.

Figure 1. Effect of grazing components on coil colonization $(\%$, mean \pm standard error; $\mathrm{n}=4)$ in Paspalum dilatatum roots: a) S: selective defoliation and P: trampling; b) P: trampling and $\mathrm{N}$ : nitrogen addition. Different letters indicate significant differences $(P<0.05)$. veces mayores que en plantas con defoliación no selectiva (cosechando toda la biomasa en pie) (Figura 1a). En cuanto a la interacción PxN, en ausencia de pisoteo el agregado de nitrógeno disminuyó $67 \%$ la colonización por ovillos, mientras que en presencia de pisoteo la adición de $\mathrm{N}$ no redujo significativamente la presencia de dichas estructuras (Figura 1b). Esta variable de la micorrización, al igual que las restantes, tampoco resultó afectada significativamente por la triple interacción SxPxN.

La intensidad de colonización por vesículas fue afectada negativamente por el pisoteo. En tratamientos sin pisoteo, la colonización por vesículas alcanzó valores de 41.02 \pm 3.54 , mientras que el pisoteo redujo la ocurrencia de estas estructuras en $17 \%$. Los restantes componentes del pastoreo y las interacciones entre ellos no afectaron el porcentaje de ocurrencia de vesículas (Tabla 1).

\section{DisCUSIÓN}

Este estudio muestra que la respuesta de la colonización micorrícica depende de una manera compleja del balance de los efectos del pisoteo, la selectividad y el agregado de $\mathrm{N}$ (esto último como una aproximación al efecto de la orina). Nuestros resultados muestran que los componentes del pastoreo presentan efectos contrapuestos y de diferente magnitud sobre los distintos aspectos de la interacción micorrícica. Dichos efectos se observaron sobre la intensidad de colonización de algunas de las estructuras fúngicas, en particular sobre los ovillos y las vesículas, mientras que los arbúsculos y la colonización total no se vieron afectados. Las diferentes respuestas registradas en arbúsculos y ovillos a los componentes del pastoreo analizados sugieren que existe un recambio en las especies de 
HMA, dado que arbúsculos y ovillos podrían corresponder a taxones diferentes de HMA (Smith and Read 2008; Hong et al. 2012). La importancia relativa de cada componente del pastoreo podría afectar el balance global de este disturbio, hecho que explicaría los efectos poco consistentes del pastoreo sobre la colonización por micorrizas arbusculares encontrados en la bibliografía (Gehring and Whitham 1994; Barto and Rillig 2010).

Paspalum dilatatum mostró niveles elevados de colonización por micorrizas arbusculares en todos los tratamientos, lo cual concuerda tanto con los antecedentes publicados (Grigera and Oesterheld 2004; García et al. 2016) así como también con observaciones realizadas en 2010 en este mismo sitio de estudio (en promedio, registramos $98 \%$ de colonización micorrícica; datos no publicados). La importante presencia de micorrizas en las raíces de $P$. dilatatum se condice con estudios previos que muestran que plantas con metabolismo fotosintético $\mathrm{C}_{4}$ presentan altos y mayores valores de colonización micorrícica en comparación con las $C_{3}$ (Lugo et al. 2003; Parodi and Pezzani 2011).

Tanto la intensidad de colonización total como por arbúsculos no fueron afectadas de manera significativa por ninguno de los componentes del pastoreo estudiados, sea de forma aislada o en interacción, lo cual coincide con estudios previos sobre otras especies de plantas (Lugo et al. 2003; Barto and Rillig 2010; Parodi and Pezzani 2011; van der Heyde et al. 2017). Lugo et al. (2003) plantean dos posibles explicaciones para la ausencia de efecto del pastoreo sobre las micorrizas. Por un lado, en suelos pobres en fósforo, como es esperable en nuestro sitio de estudio por tratarse de campo natural sin fertilizaciones previas (Hernández et al. 1995), el micelio extra-radical de los HMA se encontraría más desarrollado. Este podría actuar como un sistema estabilizador, interconectando diferentes plantas hospedantes (McNaughton and Oesterheld 1990), lo que así minimiza las diferencias entre tratamientos. Sin embargo, esto fue cuestionado ya que si bien se reconoce la importancia de la red fúngica en la transferencia de nutrientes entre plantas (van der Heijden and Horton 2009), el efecto estabilizador de dicha red es discutido por no existir evidencia clara de una relación fuente-fosa en estas transferencias (Bever et al. 2010). La otra explicación refiere a la posible existencia de crecimiento compensatorio de las plantas en respuesta al pastoreo. En este caso se postula que la defoliación provocaría un aumento de la fotosíntesis y del crecimiento de la planta; esto reestablece el balance energético y mantiene la interacción micorrícica. En este sentido, Walling y Zabinski (2006) observaron una respuesta compensatoria en términos de biomasa en dos gramíneas nativas sometidas a cortes experimentales, pero no registraron efectos sobre la presencia de HMA. En un trabajo con gramíneas nativas de los pastizales de Uruguay, Altesor et al. (2016) encontraron que una de las especies crecientes al pastoreo (Axonopus affinis) incrementó la asignación de recursos hacia sus órganos subterráneos en tratamientos que simularon la herbivoría. No se conoce aún la posible respuesta de $P$. dilatatum al pastoreo en términos de crecimiento compensatorio, aunque Bungenstab (2009) reportó que las reservas en la base de los macollos representarían una fuente de energía para compensar la pérdida de biomasa por defoliación.

Otra posible explicación sobre la ausencia de efectos de todos los componentes del pastoreo estudiados sobre la micorrización de $P$. dilatatum se relaciona con el momento en que cosechamos las plantas. La cosecha de raíces se realizó en verano, momento de mayor actividad de $P$. dilatatum, que se asocia con una mayor presencia de tejido fotosintético, lo que podría implicar que la disponibilidad de fotoasimilados sería suficiente para sustentar la interacción. En este sentido, Grigera y Oesterheld (2004) reportaron efectos negativos del pastoreo sobre la colonización micorrícica en $P$. dilatatum en invierno y primavera, pero no en verano.

Las otras estructuras participantes del intercambio entre los simbiontes, los ovillos o "coils", fueron afectados por más de un componente del pastoreo (Figura 1 a y b). El efecto de la selectividad en la remoción de biomasa sobre la colonización por ovillos dependió de la ocurrencia o no de pisoteo. En parcelas sin pisoteo y con corte selectivo de biomasa se observó una presencia de ovillos significativamente mayor que en las parcelas donde se cosechó toda la biomasa. Sin embargo, al combinarse la selectividad con el pisoteo no se detectaron efectos sobre la presencia de dichas estructuras. (Figura 1a). El tratamiento selectivo representa una presión en la remoción de biomasa de especies palatables, entre ellas P. dilatatum, un $30 \%$ mayor comparado con la biomasa removida en las parcelas en las que se cortó toda la biomasa fresca en pie (Lezama, comunicación 
personal). La colonización por ovillos aumentaría ya que tendería a compensar esas mayores pérdidas de biomasa, lo que de esta forma incrementa el abastecimiento de nutrientes hacia la planta. Si bien no hay antecedentes acerca del efecto de la remoción de biomasa vegetal sobre la presencia de ovillos, Wallace (1981) y Bayne et al. (1984) reportaron aumentos de la micorrización total debido a la defoliación. El mecanismo que podría explicar estos resultados se relaciona con una mayor producción de exudados de C hacia la rizósfera luego de la defoliación, lo que estimula el crecimiento microbiano y la mineralización de N (Bayne et al. 1984; Hamilton and Frank 2001; Ayres et al. 2004; Hamilton et al. 2008). En relación con el pisoteo, se encontraron efectos negativos sobre la microbiota del suelo en general (Li et al. 2002; McKinley et al. 2005; Hiltbrunner et al. 2012) y sobre la presencia de HMA en particular (Ritz and Young 2004; Ren et al. 2017), por lo que este factor podría estar enmascarando el efecto positivo de la selectividad sobre la colonización por ovillos.

La colonización por ovillos también fue afectada por la interacción entre el pisoteo y el agregado de N. La adición de N, que buscó simular la deposición de orina, en las parcelas sin pisoteo redujo la presencia de ovillos, mientras que al combinarse con este factor no causó efectos sobre esta variable fúngica (Figura 1b). Aunque el rol de las micorrizas en la adquisición de fósforo para las plantas es bien reconocido, recientemente se demostró que esta interacción también juega un papel fundamental en la absorción de nitrógeno (Walder et al. 2012; Hodge and Storer 2014). En las parcelas fertilizadas, el $\mathrm{N}$ mineral aumentó 40\% (Lezama and Paruelo 2016), lo que representaría una mayor disponibilidad de dicho nutriente para las plantas. La reducción en la cantidad de ovillos encontrados en las raíces de $P$. dilatatum podría indicar una dependencia menor de la planta de asociarse con los HMA para acceder a los nutrientes (Johnson et al. 2010; García et al. 2016). Los beneficios de la interacción micorrícica tienen mayor relevancia en ambientes pobres y, dado el costo que implica esta simbiosis para la planta, la colonización micorrícica tiende a disminuir en suelos con mayores contenidos de nutrientes (Tuomi et al. 2001; Collins et al. 2003). Por otra parte, la adición de $\mathrm{N}$ en presencia de pisoteo no mostró efectos sobre la abundancia de ovillos. El pisoteo podría estar causando daños mecánicos sobre las raíces y sobre el micelio extra-radicular, lo que afectaría el proceso de absorción de nutrientes, tanto por la vía directa de las plantas como por la vía fúngica (Ritz and Young 2004; Ren et al. 2017). Nuevamente, el pisoteo podría estar diluyendo el efecto negativo que tuvo la adición de $\mathrm{N}$ en las parcelas sin pisotear. De ser este el caso, el análisis del contenido de $\mathrm{N}$ de plantas de parcelas fertilizadas y sin pisoteo sería mayor que el de aquellas provenientes de parcelas fertilizadas pisoteadas.

Las diferentes respuestas que mostraron las estructuras de intercambio de los HMA en respuesta a los distintos componentes del pastoreo es uno de los resultados más destacables de nuestro trabajo. Mientras que la presencia de arbúsculos no fue afectada por ninguno de los factores analizados, los ovillos mostraron diferentes respuestas, como se detalló anteriormente. Smith y Read (2008) y Hong et al. (2012) sugieren que arbúsculos y ovillos podrían corresponder a diferentes taxones de hongos que estarían colonizando las raíces de la planta. De esta manera, los arbúsculos podrían corresponder a taxones fúngicos más resistentes a la perturbación ocasionada por el pastoreo. Si bien varias especies de HMA pueden colonizar una misma planta (van der Heijden et al. 1998; Smith and Read 2008; van der Heijden and Horton 2009), el desarrollo de uno u otro tipo de estas estructuras fúngicas está determinado tanto por el tipo de planta (Chaudhry et al. 2012) como por la identidad del simbionte fúngico (Lugo et al. 2003; Chaudhry et al. 2012; Hong et al. 2012). Los resultados del presente trabajo sugieren que aunque la colonización micorrícica total no se vio afectada por el pastoreo, pudo existir un recambio de las especies de hongos, lo cual habría alterado la composición de la comunidad micorrícica (Eom et al. 2001; Saito et al. 2004; Su and Guo 2007; Bai et al. 2013). El empleo de técnicas moleculares para identificar diferentes taxones de HMA permitirá conocer si existen cambios a nivel de la comunidad micorrícica arbuscular. van der Heyde et al. (2017) reafirman la necesidad de utilizar un set de variables a la hora de estudiar las respuestas de las micorrizas al pastoreo, con el fin de detectar cambios a nivel de composición de la comunidad fúngica, además de la intensidad de colonización. Cabe señalar que en nuestro trabajo, tanto los ovillos como los arbúsculos presentaron valores bajos; en parte, esto se podría explicar porque se realizó un único muestreo de raíces que habría limitado la 
posibilidad de encontrar estas estructuras tan efímeras.

La presencia de vesículas de HMA (estructuras de reserva de los hongos) se redujo en respuesta al pisoteo. Este disturbio físico provoca daños mecánicos sobre las raíces (Striker et al. 2006) y aumentos en la compactación del suelo (Mendoza et al. 2011; Ludvíková et al. 2014; Ren et al. 2017), lo cual podría afectar negativamente a los hongos a través de la disrupción del micelio (Ritz and Young 2004; Ren et al. 2017). Varios antecedentes reportan el efecto negativo del aumento de la compactación del suelo sobre las comunidades microbianas (Li et al. 2002; McKinley et al. 2005; Hiltbrunner et al. 2012). En el experimento sobre el cual se llevó a cabo el presente estudio, la densidad aparente del suelo se incrementó 9.2\% en las parcelas con tratamiento de pisoteo (Lezama and Paruelo 2016). Las vesículas son estructuras fúngicas de almacenamiento de lípidos; la reducción de estas estructuras debido al pastoreo podría indicar una menor reserva energética para los hongos. Por otro lado, la frecuencia de $P$. dilatatum se vio incrementada en las parcelas pisoteadas (Lezama and Paruelo 2016). El pisoteo tendría mayores perjuicios sobre otras especies de la comunidad, lo que aumentaría la disponibilidad de recursos y aliviaría la competencia sobre $P$. dilatatum, por lo que podría prescindir de la colonización micorrícica para obtener recursos.

Las interacciones micorrícicas constituyen uno de los procesos que contribuyen a aumentar la provisión de servicios ecosistémicos en áreas de pastizales, tanto de servicios de provisión (forraje y producción ganadera), como de regulación (captura de
C). Entender cómo el síndrome pastoreo afecta la interacción micorrícica es un insumo importante para el diseño de sistemas de pastoreo que maximicen la provisión de servicios. Los resultados presentados aquí permiten plantear hipótesis concretas acerca de la influencia de distintos sistemas de uso del pastizal (e.g., rotaciones, diferimiento de forraje, cargas instantáneas, combinación de especies de herbívoros, momentos de ocupación, etc.) sobre la capacidad de las gramíneas de capturar recursos y, por lo tanto, de fijar $C$. Los resultados obtenidos para $P$. dilatatum podrían reflejar el comportamiento de otras especies perennes y $\mathrm{C}_{4}$ similares a esta gramínea, aunque sería interesante incluir otras especies con diferentes atributos. Futuros trabajos deberían abordar los efectos de aplicar con mayor frecuencia $\mathrm{N}$ y otros componentes de la orina de forma de lograr una mejor simulación de este componente del pastoreo.

Agradecimientos. Los autores agradecen a la familia Macció, propietarios del establecimiento "El Relincho", por las facilidades logísticas para establecer y mantener el experimento. A Alejandra Borges del Dpto. de Estadística de la Facultad de Agronomía (UdelaR) por el asesoramiento en los análisis estadísticos; al grupo de Ecología de Pastizales (GEP) de la Facultad de Ciencias (UdelaR) por su colaboración en el trabajo de campo y al laboratorio de Microbiología de la Facultad de Agronomía (UdelaR), donde se procesaron las muestras. Enrique Jurado y dos revisores anónimos realizaron valiosas sugerencias. Este trabajo contó con el apoyo económico de ANII a través de la Beca de Iniciación INI_2010_2928.

\section{REFERENCIAS}

Altamirano, A., H. Da Silva, A. Durán, A. Echeverría, D. Panario, and R. Puentes. 1976. Clasificación de Suelos. Dirección de Suelos y Fertilizantes. Ministerio de Ganadería Agricultura y Pesca. Tomo I. Montevideo, Uruguay.

Altesor, A., E. Di Landro, H. May, and E. Ezcurra. 1998. Long-term species change in a Uruguayan grassland. Journal of Vegetation Science 9:173-80.

Altesor, A., G. Piñeiro, F. Lezama, R. B. Jackson, M. Sarasola, and J. M. Paruelo. 2006. Ecosystem changes associated with grazing in subhumid South American grasslands. Journal of Vegetation Science 17:323-332.

Altesor, A., E. Leoni, A. Guido, and J. M. Paruelo. 2016. Differential responses of three grasses to defoliation, water and light availability. Plant Ecology 217:95-104.

Ayres, E., J. Heath, M. Possell, H. I. Black, G. Kerstien, and R. D. Bardgett. 2004. Tree physiological responses to above-ground herbivory directly modify below-ground process of soil carbon and nitrogen cycling. Ecology Letters 7:469-479.

Avio, L., M. Castaldini, A. Fabiani, S. Bedini, C. Sbrana, A. Turrini, and M. Giovannetti. 2013. Impact of nitrogen fertilization and soil tillage on arbuscular mycorrhizal fungal communities in a Mediterranean agroecosystem. Soil Biology and Biochemistry 67:285-294.

Bai, G., Y. Bao, G. Du, and Y. Qi. 2013. Arbuscular mycorrhizal fungi associated with vegetation and soil parameters under rest grazing management in a desert steppe ecosystem. Mycorrhiza 23:289-301.

Barto, E. K., and M. C. Rillig. 2010. Does herbivory really suppress mycorrhiza? A meta-analysis. Journal of Ecology 
98:745-753.

Bayne, H. G., M. S. Brown, and G. J. Bethlenfalvay. 1984. Defoliation effects on mycorrhizal colonization, nitrogen fixation and photosynthesis in the Glycine-Glomus-Rhizobium symbiosis Physiologia Plantarum 62:576-80.

Bever, J. D., I. Dickie, E. Facelli, J. Facelli, J. Klironomos, M. Moora, M. Rillig, W. Stock, M. Tibbett, and M. Zobel. 2010. Rooting theories of plant community ecology in microbial interactions. Trends in Ecology and Evolution 25:468-78.

Brundrett, M., N. Bougher, B. Dell, T. Grove and N. Malajczvk. 1996. Working with mycorrhizas in forestry and agriculture. Australian Centre for International Agriculture Research. Canberra, Australia.

Bungenstab, E. 2009. Productivity, nutritive quality and utilization of Dallis Grass (Paspalum dilatatum) for beef cattle production as influenced by fertilization regime and grazing management. Tesis de doctorado. Auburn University, Alabama. USA. Pp. 104.

Chaudhry, M. S., M. Saeed, A. A. Khan, N. Sial, and M. Jamil. 2012. Morphological diversity of arbuscular mycorrhiza colonizing two aromatic grasses Veteviria zizanioides and Cymbopogon jwarancusa. Pakistan Journal of Botany $\mathbf{4 4}$ 1479-1485.

Collins, N., D. Rowland, L. Corkidi, L. Egerton-Warburton, and E. Allen. 2003. Nitrogen enrichment alters mycorrhizal allocation at five mesic to semiarid grasslands. Ecology 84:1895-1908.

Di Rienzo, J., F. Casanoves, M. Balzarini, L. González, M. Tablada, and C. Robledo. 2014. InfoStat versión 2014. Grupo InfoStat, FCA, Universidad Nacional de Córdoba, Argentina. URL: www.infostat.com.ar.

Dickson, S., F. A. Smith, and S. E. Smith. 2007. Structural differences in arbuscular mycorrhizal symbioses: more than 100 years after Gallaud, where next? Mycorrhiza 17:375-393.

Endresz, G., I. Somodi, and T. Kalapos. 2013. Arbuscular mycorrhizal colonization of roots of grass species differing in invasiveness. Community Ecology 14:67-76.

Eom, A. H., G. W. Wilson, and D. C. Hartnett. 2001. Effects of ungulates grazers on arbuscular mycorrhizal symbiosis and fungal community structure in tallgrass prairie. Mycologia 93:233-242.

García, S., F. Pezzani, A. Rodríguez, and A. del Pino. 2016. Micorrizas en gramíneas nativas: efecto de la fertilización fosfatada a largo plazo. Agrociencia 20:7-16.

Gehring, C., and T. Whitham. 1994. Interactions between aboveground herbivores and the mycorrhizal mutualists of plants. Trends in Ecology and Evolution 9:251-255.

Grigera, G., and M. Oesterheld. 2004. Mycorrhizal colonization patterns under contrasting grazing and topographic conditions in the flooding pampa (Argentina). Journal of Range Management 57:601-605.

Guo, Y., Q. Du, G. Li, Y. Ni, Z. Zhang, W. Ren, and X. Hou. 2016. Soil phosphorus fractions and arbuscular mycorrhizal fungi diversity following long-term grazing exclusion on semi-arid steppes in inner Mongolia. Geoderma 269:7990.

Hamilton, E. W., D. A. Frank, P. M. Hinchey, and T. R. Murray. 2008. Defoliation induces root exudation and triggers positive rhizospheric feedbacks in a temperate grassland. Soil Biology and Biochemistry 40:2865-2873.

Hamilton, E. W., and D. A. Frank. 2001. Can plants stimulate soil microbes and their own nutrient supply? Evidence from a grazing tolerant grass. Ecology 82:2397-2402.

Hartnett, D., and T. Wilson. 1999. Mycorrhizae influence plant community structure and diversity in tallgrass prairie. Ecology 80:1187-1195.

Hernández, J., O. Otegui, and J. P. Zamalvide. 1995. Formas y contenidos de fósforo en algunos suelos de Uruguay. Boletín de Investigaciones Facultad de Agronomía. Montevideo. No 43. Pp. 32.

Hiltbrunner, D., S. Schulze, F. Hagedorn, M. Schmidt, and S. Zimmermann. 2012. Cattle trampling alters soil properties and changes soil microbial communities in a Swiss sub-alpine pasture. Geoderma 170:369-77.

Hodge, A., and K. Storer. 2014. Arbuscular mycorrhiza and nitrogen: implications for individual plants through to ecosystems. Plant Soil 386:1-19.

Hong, J. J., Y. Park, B. Armando, K. Battarai, D. Daniels, and M. J. Harrison. 2012. Diversity of morphology and function in arbuscular mycorrhizal symbioses in Brachypodium distachyon. Planta 236:851-865.

Johnson, N. C., G. W. T. Wilson, M. A. Bowker, J. A. Wilson, and R. M. Miller. 2010. Resource Limitation Is a Driver of Local Adaptation in Mycorrhizal Symbioses. Proceedings of the National Academy of Sciences 107:2093-2098.

Koske, R., and J. Gemma. 1989. A modified procedure for staining roots to detect VA mycorrhizas. Mycological Research 92:486-505.

Lezama, F., and J. M. Paruelo. 2016. Disentangling grazing effects: trampling, defoliation and urine deposition. Applied Vegetation Science 19:557-566.

León, R. 1991. Vegetation. Pp. 380-387 en A. Soriano and R. Coupland (eds.). Natural Grasslands: introduction and Western Hemisphere. Elsevier, Amsterdam, Netherlands.

Li, C. H., B. L. Ma, and T. Q. Zhang. 2002. Soil bulk density effects on soil microbial populations and enzyme activities during the growth of maize (Zea mays L.) planted in large pots under field exposure. Canadian Journal of Soil Science 82:147-154.

Lugo, M., M. González Maza, and M. Cabello. 2003. Arbuscular mycorrhizal fungi in a mountain grassland. II: seasonal variation of colonization studied along with its relation to grazing and metabolic host type. Mycologia 93:407-415.

Ludvíková, V., V. V. Pavlů, J. Gaisler, M. Hejcman, and L. Pavlů. 2014. Long term defoliation by cattle grazing with and without trampling differently affects soil penetration resistance and plant species composition in Agrostis capillaris grassland. Agriculture, Ecosystems and Environment 197:204-11. 
Marulanda, A., R. Azcón, and J. Ruiz-Lozano 2003. Contribution of six arbuscular mycorrhizal fungal isolates to water uptake by Lactuca sativa plants under drought stress. Physiologia Plantarum 119:526-533.

McKinley, V. L., A. D. Peacock, and D. C. White. 2005. Microbial community PLFA and PHB responses to ecosystem restoration in tallgrass prairie soils. Soil Biology and Biochemistry 37:1946-1958.

McNaughton, S. J. 1984. Grazing lawns: animals in herds, plant form, and coevolution. The American Naturalist 126: 863-886.

McNaughton, S. D., and M. Oesterheld. 1990. Extramatrical mycorrhizal abundance and grass nutrition in a tropical grazing ecosystem the Serengeti National Park, Tanzania. Oikos 59:92-96.

Mendoza, R., V. Goldman, J. Rivas, V. Escudero, E. Pagani, M. Collantes, and L. Marbán. 2002. Poblaciones de hongos micorrícicos arbusculares en relación con las propiedades del suelo y de la planta hospedera en pastizales de Tierra del Fuego. Ecología Austral 12:105-116.

Mendoza, R., M. Cabello, J. Anchorena, I. García, and L. Marbán. 2011. Soil parameters and host plants associated with arbuscular mycorrhizae in the grazed Magellanic steppe of Tierra del Fuego. Agriculture, Ecosystems \& Environment 140:411-418.

Parodi, G., and F. Pezzani. 2011. Micorrizas arbusculares en dos gramíneas nativas de Uruguay en áreas con y sin pastoreo. Agrociencia 15:1-10.

Pezzani, F., C. Montaña, and R. Guevara. 2006. Associations between arbuscular mycorrhizal fungi and grasses in the successional context of a two-phase mosaic in the Chihuahuan Desert. Mycorrhiza 16:285-295.

Piippo, S., A. Markkola, E. Härmä, and J. Tuomi. 2011. Do compensatory shoot growth and mycorrhizal symbionts act as competing above and below-ground sinks after simulated grazing? Plant Ecology 212:33-42.

Porras-Alfaro, A., J. Herrera, O. D. Natvig, and R. L. Sinsabaugh. 2007. Effect of long-term nitrogen fertilization on mycorrhizal fungi associated with a dominant grass in a semiarid grassland. Plant and Soil 296:65-75.

Ren, H., W. Gui, Y. Bai, C. Stein, J. L. Rodrigues, G. W. Wilson, A. B. Cobb, Y. Zhang, and G. Yang. 2017. Long-term effects of grazing and topography on extra-radical hyphae of arbuscular mycorrhizal fungi in semi-arid grasslands. Mycorrhiza 28:117-27.

Ritz, K., and I. M. Young. 2004. Interactions between soil structure and fungi. Mycologist 18:52-59.

Ruotsalainen, A., and A. Eskelinen. 2011. Root fungal symbionts interact with mammalian herbivory, soil nutrient availability and specific hábitat conditions. Oecologia 166:807-817.

Saito, K., Y. Suyama, S. Sato, and K. Sugawara. 2004. Defoliation effects on the community structure of arbuscular mycorrhizal fungi based on 18s rADN sequences. Mycorrhiza 14:363-373.

Schausberger, P., S. Peneder, S. Jürschik, and D. Hoffmann. 2012. Mycorrhiza changes plant volatiles to attract spider mite enemies. Functional Ecology 26:441-449.

Smith, S. E., and D. J. Read. 2008. Mycorrhizal Simbiosis. $3^{\text {rd }}$ edition. Academic Press, New York, USA.

Smith, S. E., and F. A. Smith. 2011. Roles of arbuscular mycorrhizas in plant nutrition and growth: new paradigms from cellular to ecosystem scales. Annual Review of Plant Biology 62:227-50.

Stillwell, M. A., and R. G. Woodmansee. 1981. Chemical transformation of urea-nitrogen and movement of nitrogen in shortgrass prairie soil. Soil Science Society of America Journal 45:893-898.

Striker, G., P. Insausti, A. Grimoldi, and R. León. 2006. Root strength and trampling tolerance in the grass Paspalum dilatatum and the dicot Lotus glaber in flooded soil. Functional Ecology 20:4-10.

$\mathrm{Su}, \mathrm{Y}$. , and L. Guo. 2007. Arbuscular mycorrhiza fungi in non-grazed, restored and over-grazed grassland in the Inner Mongolia steppe. Mycorrhiza 17:689-693.

Torres, Y., C. Busso, O. Montenegro, L. Ithurrart, H. Giorgetti, G. Rodríguez, D. Betivenga, R. Brevedan, O. Fernández, M. Mujica, S. Baioni, J. Entío, M. N. Fioreti, and G. Tucat. 2011. Defoliation effects on the arbuscular mycorrhizas of ten perennial grass genotypes in arid Patagonia, Argentina. Applied Soil Ecology 49:208-214.

Tuomi, J., M. M. Kytöviita, and R. Härdling. 2001. Cost efficiency of nutrient acquisition and the advantage of mycorrhizal symbiosis for the host plant. Oikos 92:62-70.

van der Heijden, M., J. Klironomos, M. Ursi, P. Moutoglis, R. Stritwolf-Engel, T. Boller, A. Wiemken, and I. Sanders. 1998. Mycorrhizal fungi diversity determines plant biodiversity, ecosystem variability and productivity. Nature 395:69-72.

van der Heijden, M, and T. R. Horton. 2009. Socialism in soil? The importance of mycorrhizal fungal networks for facilitation in natural ecosystems. Journal of Ecology 97:1139-1150.

van der Heyde, M., J. A. Bennett, J. Pither, and M. Hart. 2017. Long term effects of grazing on arbuscular mycorrhizal fungi. Agriculture, Ecosystems and Environment 243:27-33.

Vierheilig, H., A. P. Coughlan, U. Wiss, and Y. Piché. 1998. Ink and vinegar, a simple staining technique for arbuscularmycorrhizal fungi. Applied Environmental Microbiology 64:5004-5007.

Walder, F., H. Niemann, M. Natarajan, M. Lehmann, T. Boller, and A. Wiemken. 2012. Mycorrhizal networks: common goods of plants shared under unequal terms of trade. Plant Physiology 159:789-797.

Wallace, L. L. 1981. Growth, morphology and gas exchange of mycorrhizal and nonmycorrhizal Panicum coloratum L., $\mathrm{a} \mathrm{C}_{4}$ grass species, under different clipping and fertilization regimes. Oecologia 49:272-278.

Walling, S., and C. A. Zabinski. 2006. Defoliation effects on arbuscular mycorrhizae and plant growth of two native bunchgrasses and an invasive forb. Applied Soil Ecology 32:111-117. 